\title{
Counting Geodesics, Teichmüller Space, and Random Hyperbolic Surfaces
}

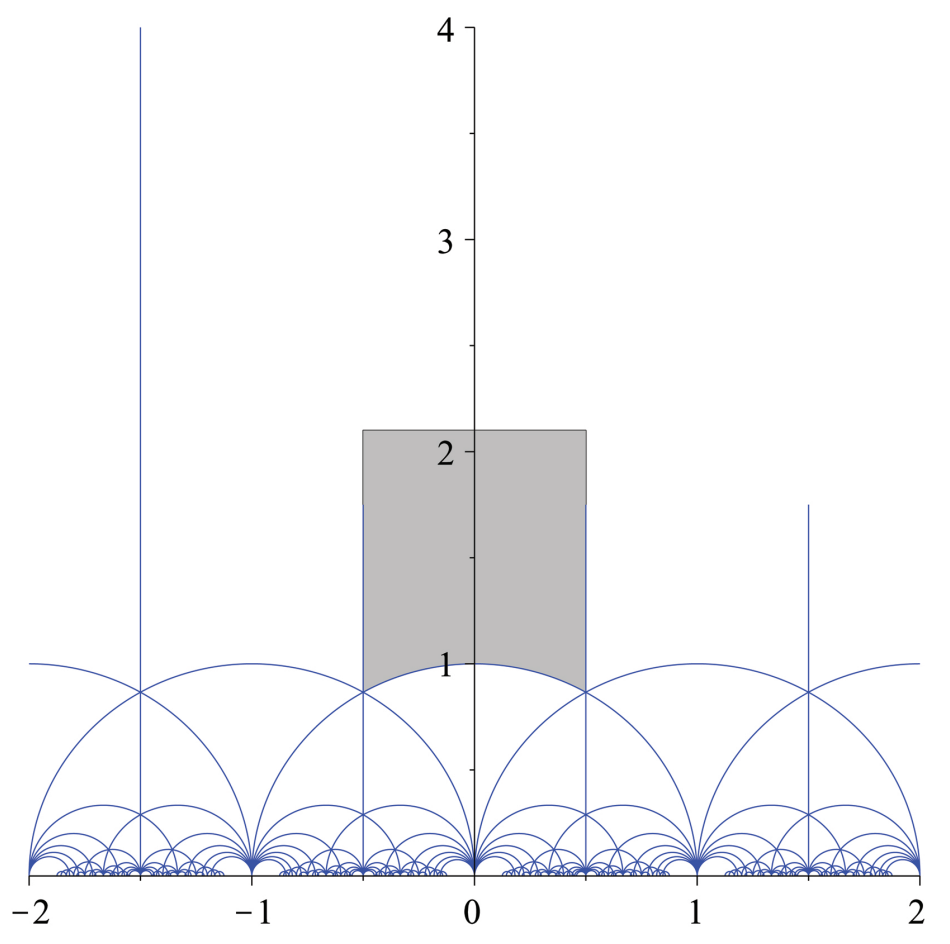

\section{Scott A. Wolpert}

\section{Introduction}

The study of geodesics provides a theme for understanding hyperbolic metrics on finite-area surfaces, as well as the geometry of the moduli space of Riemann surfaces. We begin with the fundamentals of the Teichmüller theory of hyperbolic surfaces. We relate how formulas in hyperbolic geometry correspond to formulas for the Teichmüller space and moduli space. Then we describe how

Scott A. Wolpert is a professor emeritus of mathematics at the University of Maryland. His email address is saw@math. umd. edu.

Communicated by Notices Associate Editor Chikako Mese.

For permission to reprint this article, please contact:

reprint-permission@ams.org.

DOI: https://doi.org/10.1090/noti2386
Thurston's random geodesic metric generalizes to the pressure metric in higher Teichmüller theory and how Mirzakhani's recursive integration scheme is a tool for understanding random finite-area hyperbolic surfaces. Our informal exposition does not attempt a summary of a historical development of the subject. Too many important results are not discussed and the development of ideas is greatly simplified. Only limited references are provided. For further resources the reader may explore McMullen's Expositions and Course Notes at the bottom of his Publications. A general introduction to the present material is provided in "Riemann surfaces, dynamics and geometry"all available on people.math.harvard.edu/ ctm/. The reader may also explore the author's CBMS and PCMI 


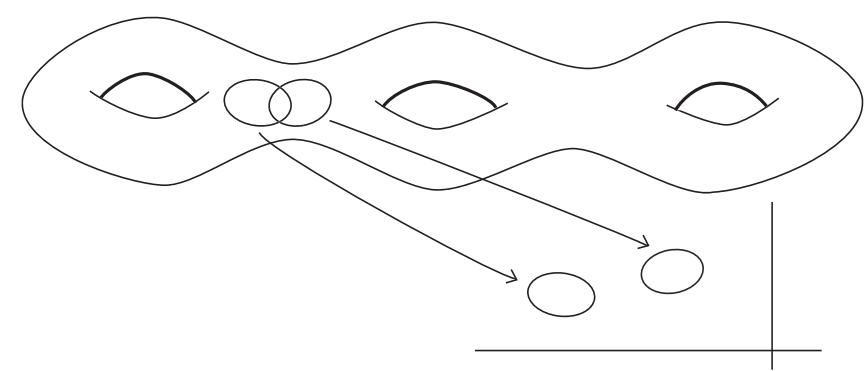

Figure 1. Charts for a genus 3 surface.

lectures [11, 12]. And a valuable exercise is to use AMS MATHSCINET to check the publications that refer to the references here-a reverse search.

\section{Hyperbolic Geometry and Riemann Surfaces}

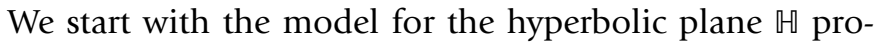
vided by the upper half-plane $\{z \mid \Im z>0\} \subset \mathbb{C}$ with the Riemannian metric $(|d z| / \Im z)^{2}[3,5]$. The group of orientationpreserving isometries is given by $\operatorname{PSL}(2 ; \mathbb{R})$ acting by fractional linear transformations on $\mathbb{C}$ preserving the upper half-plane. The maximal geodesics are the semicircles with centers on the real axis and the vertical half-lines. A Riemann surface $R$ is prescribed by a covering of a topological space by an atlas of charts $\left\{\left(U_{\alpha}, z_{\alpha}\right)\right\}$ with $z_{\alpha}$ maps to the complex plane $\mathbb{C}$ such that the overlap maps $z_{\beta} \circ z_{\alpha}^{-1}$ are biholomorphic. A Riemannian metric for a surface is prescribed by specifying positive coefficients $\left\{\left(U_{\alpha}, g_{\alpha}\right)\right\}$ such that $g_{\alpha}=g_{\beta}\left|\frac{d z_{\beta}}{d z_{\alpha}}\right|^{2}$. Alternately, a hyperbolic metric can be specified by special charts- $\left\{\left(U_{\alpha}, w_{\alpha}\right)\right\}$ with $w_{\alpha}$ maps to the hyperbolic plane $\mathbb{U}$ with $w_{\beta} \circ w_{\alpha}^{-1}$ local isometries. A surface with metric is also described by gluing (attaching by isometries) shapes from a model space. For example a flat torus is given by identifying opposite sides of a Euclidean parallelogram.

The classical modular surface is given by gluing the domain $\mathcal{D}=\left\{z|| \Re z\left|\leq \frac{1}{2},\right| z \mid \geq 1\right\}$. The hyperbolic triangle $\mathcal{D}$ has vertices at $\rho$ and $\rho^{2}, \rho=e^{i \pi / 3}$, and an ideal vertex with zero angle at infinity. The vertical sides are glued by the translation $T: z \rightarrow z+1$. The semicircle segment $|z|=1, \Re z \leq 0$ is glued to the semicircle segment $|z|=1, \Re z \geq 0$ by the negative inversion $S: z \rightarrow-1 / z$. A neighborhood of the image of $\rho$ in the quotient space is alternately described by $\mathcal{D} \cup T \mathcal{D}$ with $S$ identifying the semicircles $|z|=1$ and $|z-1|=1$. The points $\rho$ and $\rho^{2}$ correspond to a cone point in the quotient space. The total angle around the point is $2 \pi / 3$. A neighborhood of the image of infinity in the quotient space is a cusp-the point infinity is at infinite distance. The area of $\mathcal{D}$ is the angle defect $\pi / 3$. The matrices $T= \pm\left(\begin{array}{ll}1 & 1 \\ 0 & 1\end{array}\right)$ and $S= \pm\left(\begin{array}{cc}0 & -1 \\ 1 & 0\end{array}\right)$ generate the classical modular group $\operatorname{PSL}(2 ; \mathbb{Z})$ - the quotient of the group of $2 \times 2$ integer matrices with determinant 1 by $\pm\left(\begin{array}{ll}1 & 0 \\ 0 & 1\end{array}\right)$. The domain $\mathcal{D}$ is a fundamental domain for the action of the modular group on the hyperbolic plane. The

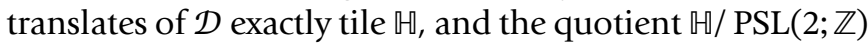
is the identification space of $\mathcal{D}$.

Convexity is a basic feature of hyperbolic geometry. Accordingly a closed loop on a hyperbolic surface can be pulled taut to form a closed geodesic. Each nontrivial, noncuspidal, free homotopy class contains a unique closed geodesic that is length minimizing. Similarly, each free homotopy class of arcs (with sliding endpoints) between a pair of closed geodesics contains a unique geodesic segment. The segment realizes the distance. Geodesics in hyperbolic geometry exhibit very special behaviors. Following a result of Birman-Series, Sapir showed that on a finite-area surface the collection of geodesics with bounds on the number of self-intersections is contained in a closed set of Hausdorff dimension one and Lebesgue measure zero. Geodesics with less than maximal self-intersections lie only in narrow channels on a surface. By total contrast, the collection of all closed possibly self-intersecting geodesics is uniformly distributed. Given $\epsilon>0$ small, there is an $L$ large such that given a tangent vector $v$, the total length of segments of closed geodesics of length at most $L$ with tangents $\epsilon$-close to $v$ is positive and approximately independent of $v$. In fact, after normalization the total length of segments close to a given tangent vector tends to the uniform distribution in $v$ as $L$ tends to infinity. In effect closed geodesics almost uniformly almost pass through each point with almost each tangent direction. Consequently we can contemplate the behavior of the random closed geodesic. A starter question is to count by length the number of closed geodesics. In analogy to the prime number theorem, Delsarte, Huber, and Selberg each introduced a trace formula to perform a count. For a finite-area hyperbolic surface the count is exponential. The number of closed geodesics of length at most $L$ is asymptotic to $e^{L} / L$. We will see that the count is polynomial when the number of self-intersections is restricted.

By the Uniformization Theorem, a surface $R$ with complete hyperbolic metric is described as a quotient $\mathbb{H} / \Gamma$, where $\Gamma$ is a subgroup of $\operatorname{PSL}(2 ; \mathbb{R})$. The group $\Gamma$ is isomorphic to the fundamental group of $R$ and acts on $\mathbb{H}$ as the group of deck transformations. The hyperbolic plane is tiled by $\Gamma$-translates of a fundamental domain $\mathcal{D}$, a finitesided polygon. If $R$ has genus $g$ and $n$ cusps, the Euler characteristic is negative and the area of $R$ is $2 \pi(2 g-2+n)$.

\section{Teichmüller Spaces}

Fenchel-Nielsen introduced a synthetic geometric tailoring construction for prescribing a hyperbolic surface $R$ $[3,5,11]$. The construction begins with the observation that in the hyperbolic plane right-angled hexagons $\mathcal{H}$ are specified by giving the lengths of alternate sides as positive real numbers. Side lengths can also have the ideal value 
zero and then the adjacent sides of the hexagon describe a cusp as for the PSL $(2 ; \mathbb{Z})$ fundamental domain. Given a right hexagon $\mathcal{H}$ with side lengths $\ell_{1} / 2, \ell_{2} / 2$, and $\ell_{3} / 2$, then double $\mathcal{H}$ across the alternate sides to obtain a pair of pants $\mathcal{P}$-a surface with the topology of a sphere minus three disjoint discs. The pants $\mathcal{P}$ has geodesic boundaries since the double across one boundary of a quarter-plane is a half-plane. The $\mathcal{P}$ boundary lengths are $\ell_{1}, \ell_{2}$, and $\ell_{3}$ and the area is $2 \pi$. Also attaching half-planes is a local construction. Accordingly pants $\mathcal{P}_{1}$ and $\mathcal{P}_{2}$ with a common boundary length $\ell$ can be attached across the common boundary to form a hyperbolic surface with four geodesic boundaries and the topology of a sphere minus four disjoint discs. Remaining boundaries of equal length can also be identified. For example starting with two copies of a pair of pants $\mathcal{P}$, the boundaries of equal lengths can be identified in pairs to form a compact surface of genus 2 . In general a surface of genus $g$ with $n$ cusps is tailored by starting with $2 g-2+n$ pairs of pants and identifying in pairs boundaries of equal lengths. The collection of seams, a collection of $3 g-3+n$ disjoint simple (no self-intersections) closed geodesics, is called a pants decomposition.

What are the parameters for constructing a surface by tailoring pants? First there are the $3 g-3+n$ seam lengths, values in $\mathbb{R}^{+}$. Second there is a twist parameter for each seam. A pair of pants has an equator reflection given by interchanging hexagons. The fixed set of a reflection is the three geodesic segments between boundary components. Since boundaries are circles, pants can be assembled with an arbitrary relative displacement between boundaries. The displacement between the equators on the two sides of a seam is a parameter. The Fenchel-Nielsen twist parameter $\tau$ is the displacement between equators measured in hyperbolic length units. The twist parameter similar to angle measure takes values in $\mathbb{R}$. Tailoring includes labeling pants and loops-the instructions also prescribe a basis for the fundamental group of a surface. Teichmüller space is the space of hyperbolic surfaces with a reference isomorphism for the fundamental groups modulo an equivalence relation.

Fenchel-Nielsen Theorem. The length-twist parameters

$$
\prod_{j=1}^{3 g-3+n}\left(e_{j}, \tau_{j}\right) \in\left(\mathbb{R}^{+} \times \mathbb{R}\right)^{3 g-3+n}
$$

are real-analytic coordinates for Teichmüller space.

Different pants decompositions provide different global coordinates. In the next section we see that a completion of Teichmüller space is prescribed by allowing collections of pants lengths to vanish.

How many ways can an individual surface be tailored? A simple redundancy is given by adding the seam length to a twist parameter $\tau \rightarrow \tau+\ell$. This parameter alteration

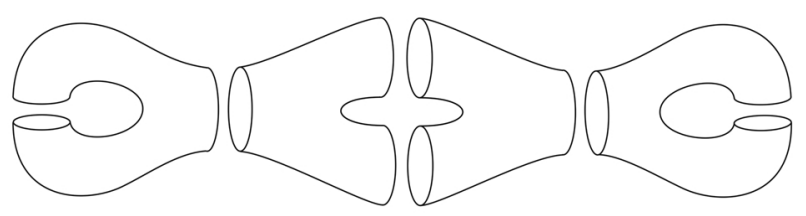

Figure 2. Constructing a genus 3 surface from pants.

corresponds to applying a homeomorphism, a Dehn twist, that is a full twist in a neighborhood of the seam curve and the identity otherwise. More generally new pants decompositions for a fixed surface are given by applying homeomorphisms. Even more generally there are pants decompositions not related by homeomorphisms. For example in genus two, modulo homeomorphisms, there are two distinct decompositions: one with no separating curves and one with a single separating curve. Bollobás found the asymptotic count of distinct pants decompositions-the leading term is $(6 g-6) ! /(3 g-3) !(2 g-2) !$. By definition decompositions related by homeomorphisms are related by the mapping class group $M C G$ - the group of orientationpreserving homeomorphisms of a surface modulo its subgroup of homeomorphisms isotopic to the identity. The group acts transitively on the reference isomorphisms for the points of the Teichmüller space $\mathcal{T}$. The action of $M C G$ on $\mathcal{T}$ is properly discontinuous. By a theorem of Dehn, $M C G$ is generated by Dehn twists. The quotient $\mathcal{T} / M C G$ is the moduli space $\mathcal{M}$ of Riemann surfaces-the space of distinct Riemann surfaces.

\section{Moduli Spaces}

A Fenchel-Nielsen tailoring description of $\mathcal{M}$ is given by a description of an $M C G$ fundamental domain in $\mathcal{T}$. By all indications a general description is expected to be intractable. For simple applications an estimate of Buser and Parlier suffices. Every closed hyperbolic surface has a pants decomposition with all seams of length at most $4 \pi(g-1)+4 \log (4 g-2)+\operatorname{arcsinh} 1$.

A basic deformation of hyperbolic surfaces is pinching collars-a taffy pulling deformation. Given a simple closed geodesic $\gamma$ of length $\ell$, the collar about $\gamma$ is the tubular neighborhood $\mathcal{C}=\{p \mid d(p, \gamma) \leq w\}$ for $w=\operatorname{arcsinh}(1 / \sinh (\ell / 2))$ with the hyperbolic metric on $\mathcal{C}$ isometric to $d \rho^{2}+\ell^{2} \cosh ^{2} \rho d t^{2}$. The collar lemma provides that simple geodesics have collars and disjoint simple geodesics have disjoint collars. Since $w$ is large for $\ell$ small, it follows that short simple geodesics are disjoint. From an additional observation it follows that short geodesics are necessarily simple. The diameter of a containing surface is at least the collar half-width $w$. Taffy snaps into two pieces after extreme pulling. The limit of collars with core lengths $\ell$ tending to zero is a pair of cusps. What are the possible limits for a sequence of hyperbolic 


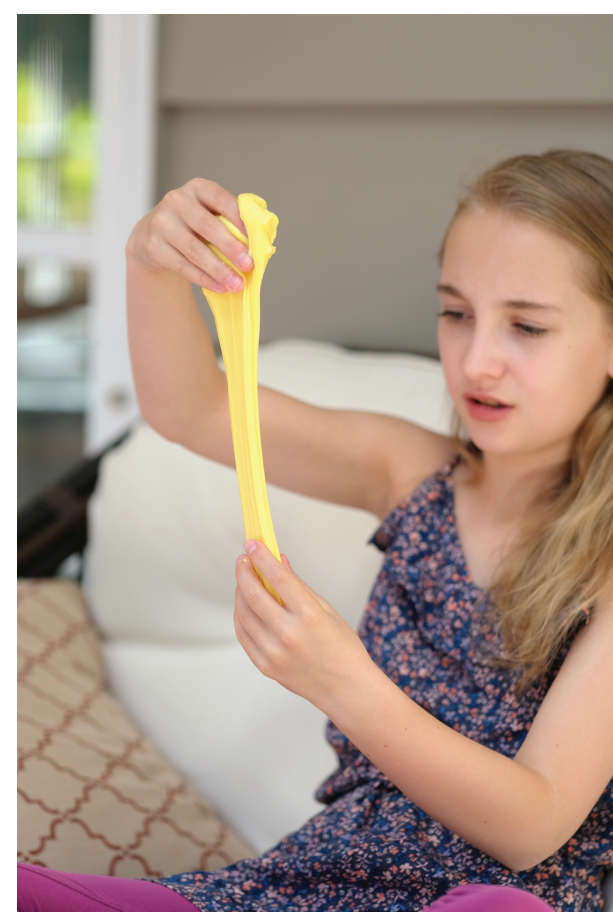

Figure 3. Pulling taffy.

surfaces? The first consideration is whether there is a positive lower bound for the lengths of closed geodesics.

Mumford Compactness Theorem. Given $\ell_{0}$ positive, the subset of the moduli space $\mathcal{M}$ of hyperbolic surfaces with all closed geodesics of length at least $\ell_{0}$ is compact.

The result can be extended. If collars about short geodesics are removed from hyperbolic surfaces, then the collection of metric spaces is precompact in the GromovHausdorff topology. When pulling taffy, the ends deform only a bounded amount. The complements in hyperbolic surfaces of the collars about short geodesics only vary a bounded amount.

The considerations indicate that $\mathcal{M}$ is compactified by simply permitting collars to limit to pairs of cusps. In particular given Fenchel-Nielsen coordinates $\left(\ell_{j}, \tau_{j}\right)$, a boundary space is added to Teichmüller space by permitting length parameters to take the 0 -value, describing cusps for the corresponding pants and hexagons. The twist parameter is undefined for zero length. Paired cusps are always included as elements of a pants decomposition. A surface with paired cusps and a fundamental group reference isomorphism is described by multiple pants decompositions-giving rise to an equivalence relation for boundary spaces.

Definition 1. The augmented Teichmüller space $\overline{\mathcal{T}}$ consists of the Teichmüller space with all boundary spaces for all pants decompositions modulo equivalence.

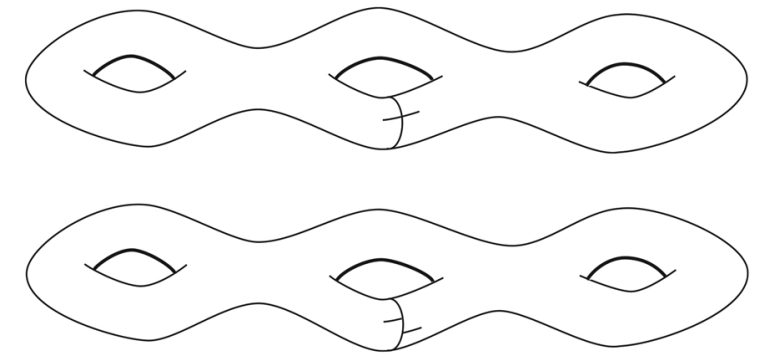

Figure 4. A Fenchel-Nielsen twist deformation.

The mapping class group acts on $\overline{\mathcal{T}}$, and the quotient $\overline{\mathcal{T}} / M C G$ is compact and homeomorphic to the DeligneMumford stable curve compactification of the moduli space $\mathcal{M}$. Fenchel-Nielsen coordinates $(\ell, \tau)$ are analogous to polar coordinates $(r, \theta)$ for the universal cover of $\mathbb{R}^{2} \backslash\{0\}$; accordingly $\overline{\mathcal{T}}$ is not locally compact. We will see that $\overline{\mathcal{T}}$ has a direct relationship to the Weil-Petersson (WP) metric.

\section{Weil-Petersson Geometry}

How do we describe the geometry of Teichmüller space $\mathcal{T}$ ? We start with the twist vector fields and length functions coming from Fenchel-Nielsen coordinates [3, 5, 11]. Given the free homotopy class of a simple closed loop $\alpha$, the reference isomorphisms of fundamental groups determine loops and upon pulling taut geodesics for each point of $\mathcal{T}$. The twist vector field $t_{\alpha}$, an infinitesimal deformation for each point of $\mathcal{T}$, is defined as the infinitesimal variation of the twist parameter for $\alpha$. The geodesic-length function $\ell_{\alpha}$ is defined as the length of the geodesic $\alpha$ on the surface represented by the point of $\mathcal{T}$. For simple closed geodesics on each surface, multiples of the twist tangents, respectively, multiples of the geodesic-length differentials, are dense in the tangent space, respectively, cotangent space, of $\mathcal{T}$. We use twists and lengths to describe the geometry.

We begin with Riera's formula for the pairing of geodesic-length gradients-the formula can serve as a definition for the pairing. Introduce the positive function $G(\ell)=\cosh \ell \log ((\cosh \ell+1) /(\cosh \ell-1))-2$.

Theorem 2. For $\alpha, \beta$ disjoint simple closed geodesics, the WeilPetersson pairing is given as

$$
\left\langle\operatorname{grad} \ell_{\alpha}, \operatorname{grad} \ell_{\beta}\right\rangle=\frac{2}{\pi}\left(e_{\alpha} \delta_{\alpha \beta}+\sum_{\gamma} G\left(e_{\gamma}\right)\right)
$$

for the Kronecker delta $\delta_{*}$ and the sum over the set of minimal length geodesic arcs connecting $\alpha$ to $\beta$.

An analysis involving the geometry of collars shows for $\ell_{\alpha}$ small that $d \ell_{\alpha} / d s=\left\|\operatorname{grad} \ell_{\alpha}\right\| \approx\left(2 \ell_{\alpha} / \pi\right)^{1 / 2}$; consequently $\int d s=\int_{0}^{1}\left\|\operatorname{grad} \ell_{\alpha}\right\|^{-1} d \ell_{\alpha}$ is finite and collars can 
be pinched in finite WP time-the metric is incomplete. In line with the moduli space being compactified by permitting collars to limit to cusp pairs, Masur found that the augmented Teichmüller space $\overline{\mathcal{T}}$ is the WP completion of $\mathcal{T}$.

There is a rich symplectic geometry of twists and lengths corresponding to the hyperbolic geometry of surfaces. The WP metric is Kähler with symplectic form $\omega-$ a closed nondegenerate 2-form measuring area of 2-planes.

Theorem 3 ([11]). Let $\alpha, \beta$ be simple closed geodesics. Twists and lengths satisfy the duality relations

$$
2 t_{\alpha}=i \operatorname{grad} \ell_{\alpha} \text { and } 2 \omega\left(, t_{\alpha}\right)=d \ell_{\alpha} .
$$

The twist derivatives of lengths are given as

$$
\begin{gathered}
t_{\alpha} \ell_{\beta}=\omega\left(t_{\alpha}, t_{\beta}\right)=\sum_{p \in \alpha \# \beta} \cos \theta_{p}, \\
t_{\alpha} t_{\alpha} \ell_{\beta}=\sum_{(p, q) \in \alpha \# \beta \times \alpha \# \beta} \frac{e^{\ell_{1}}+e^{\ell_{2}}}{2\left(e^{\ell_{\beta}}-1\right)} \sin \theta_{p} \sin \theta_{q},
\end{gathered}
$$

where angles are measured from the first to the second geodesic and $\ell_{1}, \ell_{2}$ are the distances along $\beta$ between $p$ and $q$.

The cosine formula is valid even when the Teichmüller space is a single point. In particular the sum of cosines is zero for any two closed geodesics on the modular surface $\mathbb{H} / \operatorname{PSL}(2 ; \mathbb{Z})$. The summands for the sine formula are positive. A consequence is that geodesic lengths are convex functions along twisting paths-an ingredient in Kerckhoff's proof of Nielsen Realization: every finite $M C G$ subgroup has a fixed point. A third consequence is that $\omega$ is invariant under each twist flow. Knowing that twists are dense in each tangent space suggests that $\omega$ is highly symmetric.

Theorem $4([11,13])$. For Fenchel-Nielsen coordinates, the WP symplectic form is

$$
\omega=\frac{1}{2} \sum_{j} d \ell_{j} \wedge d \tau_{j}
$$

This simple formula is an ingredient in Mirzakhani's integration scheme.

Convexity is also a consideration for understanding WP geodesics.

Theorem 5 ([11]). Geodesic-length functions are strictly convex along WP geodesics.

Yamada combined geodesic convexity and an analysis of the metric near boundary spaces to show that $\overline{\mathcal{T}}$ is $C A T(0)$. A simply connected complete metric space is $C A T(0)$ provided distance is realized by unique lengthminimizing paths and provided triangles have Euclidean comparisons. Chords for triangles in $\overline{\mathcal{T}}$ are at most as long as corresponding chords for Euclidean triangles with the corresponding side lengths-a condition for a nonpositively curved space. The intersection pattern of boundary spaces can be combined with the $C A T(0)$ geometry to establish the Masur-Wolf result: the $M C G$ is the full group of WP isometries. Daskalopoulos-Wentworth applied the $C A T(0)$ geometry to analyze the action of the elements of $M C G$.

\section{The Random Geodesic Metric}

The geodesic flow on the set of unit tangent vectors of a manifold is defined by following a geodesic with a given initial tangent. For negatively curved manifolds the flow is topologically transitive-has a dense orbit. The flow is also Anosov-the bundle of unit tangent vectors decomposes into three invariant subspaces: one on which the flow is expanding, one on which the negative time flow is expanding, and the one-dimensional bundle of tangents to the flow. The closed orbits of the flow are the closed geodesics with their tangents. For hyperbolic surfaces Thurston observed that the statistics of variations of geodesic lengths define a metric on Teichmüller space. More recently from the work of a collection of authors, we know the Thermodynamic Formalism can be used to measure the difference of Anosov-type flows and gives rise to a seminorm, the pressure metric, on certain deformation spaces.

Thurston's definition is based on the properties of uniformly distributed orbits and on properties of twist derivatives.

Definition 6. Thurston's random geodesic metric for Teichmüller space is defined as

$$
\left\|t_{\alpha}\right\|_{T}^{2}=\lim _{L \rightarrow \infty} \frac{\sum_{\ell_{\beta} \leq L} t_{\alpha}^{2} \ell_{\beta}}{\sum_{\ell_{\beta} \leq L} \ell_{\beta}} .
$$

Observations are needed. Uniform distribution provides that the number of intersections $\alpha \# \bigcup_{\ell_{\beta} \leq L} \beta$ is proportional to the length sum itself and the values $\theta_{p}$ are almost uniformly distributed in $[0, \pi]$. An analysis shows the limit exists and is positive. By uniform distribution of angle values, the limit of $\sum_{\ell_{\beta} \leq L} t_{\alpha} \ell_{\beta} / \sum_{\ell_{\beta} \leq L} \ell_{\beta}$ is the integral $\int_{0}^{\pi} \cos \theta d \theta$. The first twist derivative vanishes and consequently the second twist derivative defines a quadratic form on tangent vectors. Additional considerations show that the quadratic form is positive definite. We applied an analysis of the variation of the measures on the spaces of geodesics to conclude the following.

Theorem 7 ([14]). Thurston's random geodesic metric is a multiple of the Weil-Petersson metric

$$
\langle,\rangle_{W P}=3 \pi(g-1)\langle,\rangle_{T} \text {. }
$$

The trigonometric expressions for the second twist derivative converge to the trigonometric expressions in $\mathrm{Ri}$ era's formula. 
The Thermodynamic Formalism provides a general framework for using the statistics of periods of closed orbits to define a seminorm $[1,2]$. Let $\varphi_{t}$ be a topologically transitive Anosov-type flow on a space $X$. For a $\varphi_{t}$-orbit let $\ell(a)$ be its period and consider the count

$$
R_{L}=\{\text { a closed orbit } \mid \ell(a) \leq L\} .
$$

Bowen observed that the topological entropy is the exponential growth rate of the count:

$$
h(\varphi)=\limsup _{L \rightarrow \infty} \frac{\log \# R_{L}}{L} .
$$

If $m: X \rightarrow \mathbb{R}$ is a Hölder function and $a$ is a closed orbit, define the period relative to $m$ as

$$
\ell_{m}(a)=\int_{0}^{\ell(a)} m\left(\varphi_{s}(x)\right) d s
$$

Bowen-Ruelle and Sambarino consider the pressure of $m$ as given by

$$
\mathbf{P}(m)=\limsup _{l \rightarrow \infty} \frac{1}{L} \log \left(\sum_{a \in R_{L}} e^{\ell_{m}(a)}\right) .
$$

The above period integral is the model for defining a modified time scale for the flow. Given a positive Hölder function $f$ on $X$, there is a reparameterized flow with periods $\ell_{f}(a)$ and reparameterization of time

$$
\kappa_{f}(x, t)=\int_{0}^{t} f\left(\varphi_{s}(x)\right) d s .
$$

The integral of $f$ along orbits is the modified elapsed time. The flow with the new time scale $\varphi_{t}^{f}$ is defined by

$$
\varphi_{\kappa_{f}(x, t)}^{f}(x)=\varphi_{t}(x) .
$$

In this setting Ruelle showed that the pressure is a convex function-in particular for Hölder functions $f$ and $m$,

$$
\left.\frac{\partial^{2}}{\partial t^{2}}\right|_{t=0} \mathbf{P}(f+t m) \geq 0 .
$$

Sambarino showed that the topological entropy is precisely the scale factor to give pressure zero-in particular for $\varphi$ a topologically transitive Anosov-type flow $\mathbf{P}(-h f)=$ 0 if and only if $h=h\left(\varphi^{f}\right)$.

Following McMullen a pressure seminorm is defined on the space of pressure zero Hölder functions. In particular for $f$ in $\mathcal{P}$ and $m$ a tangent to $\mathcal{P}$, define the pressure seminorm at $f$ as

$$
\|m\|_{\mathbf{P}}^{2}=-\frac{\left.\frac{\partial^{2}}{\partial t^{2}}\right|_{t=0} \mathbf{P}(f+t m)}{\left.\frac{\partial}{\partial t}\right|_{t=0} \mathbf{P}(f+t f)} .
$$

Ruelle and Parry-Pollicott showed that the pressure seminorm characterizes the variations of orbit periods.
Theorem 8. Let $\varphi$ be a topologically transitive Anosov-type flow and $m$ a tangent vector to $\mathcal{P}$. Then

$$
\|m\|_{\mathbf{P}}=0 \text { if and only if } \ell_{m}(a)=0
$$

for every closed orbit.

Establishing the relation to Thurston's random geodesic metric involves an intersection number introduced by Bonahon. The renormalized intersection number for two positive Hölder functions is

$$
\mathbf{J}\left(f_{1}, f_{2}\right)=\frac{h\left(f_{2}\right)}{h\left(f_{1}\right)} \lim _{L \rightarrow \infty} \frac{1}{\# R_{L}\left(f_{1}\right)} \sum_{a \in R_{L}\left(f_{1}\right)} \frac{\ell_{f_{2}}(a)}{\ell_{f_{1}}(a)} .
$$

Proposition 9 ([1,2]). Let $\varphi$ be a topologically transitive Anosov-type flow and $f_{t}$ a smooth family of positive Hölder functions with the pressure zero family $\Phi(t)=-h\left(\varphi^{f_{t}}\right) f_{t}$. Then

$$
\left\|\dot{\Phi}_{0}\right\|_{\mathbf{P}}^{2}=\left.\frac{\partial^{2}}{\partial t^{2}}\right|_{t=0} \mathbf{J}\left(f_{0}, f_{t}\right) .
$$

The Thermodynamic Formalism is suited for considering representation spaces. Teichmüller space is alternately described as the space of discrete faithful representations of a compact surface fundamental group into $\operatorname{PSL}(2 ; \mathbb{R})$ modulo conjugation- $\operatorname{Hom}_{d f}\left(\pi_{1}, \operatorname{PSL}(2 ; \mathbb{R})\right) / \operatorname{PSL}(2 ; \mathbb{R})$. Geodesic lengths and traces are simply related by $2 \cosh \ell_{\alpha} / 2=|\operatorname{tr} A|$. The deck transformations of a hyperbolic surface describe a $\operatorname{PSL}(2 ; \mathbb{R})$ representation. For hyperbolic surfaces the topological entropy is the constant 1. Theorem 8 corresponds to the classical fact that an infinitesimal variation is trivial exactly when all length variations vanish.

Bridgeman-Canary-Labourie-Sambarino showed that there is an analytic mapping from $\mathcal{T}$ to the space $\mathcal{P}$ of pressure zero reparameterizations for a hyperbolic surface geodesic flow $[1,2]$. Then Bonahon's setting for geodesic currents is used to show that the second derivative of the renormalized intersection number is Thurston's random geodesic metric-the Weil-Petersson metric.

Bridgeman-Canary-Labourie-Sambarino developed the Thermodynamic Formalism for Hitchin representations into $\operatorname{PSL}(d ; \mathbb{R}), d \geq 3$. The group $\operatorname{SL}(2 ; \mathbb{R})$ acts on indeterminates $x, y$ by $(x, y)\left(\begin{array}{ll}a & b \\ c & d\end{array}\right)=(a x+c y, b x+d y)$. Accordingly $\operatorname{SL}(2 ; \mathbb{R})$ acts on $\mathcal{S}_{d}$, the homogeneous polynomials of degree $d$ in the variables $x, y$. The action is irreducible-an $\mathrm{SL}(2 ; \mathbb{R})$ invariant subspace of $\mathcal{S}_{d}$ is either $\{0\}$ or $\mathcal{S}_{d}$. Relative to the basis $\left\{x^{d-1}, x^{d-2} y, \ldots, x y^{d-2}, y^{d-1}\right\}$ the action of $A \in \mathrm{SL}(2 ; \mathbb{R})$ defines an element $\tau_{d}(A) \in \mathrm{SL}(d ; \mathbb{R})$ the mapping $\tau_{d}$ is a group homomorphism. Accordingly for a compact surface $S$ and representation $\rho: \pi_{1}(S) \rightarrow$ $\operatorname{PSL}(2 ; \mathbb{R})$ there is an associated representation $\tau_{d}(\rho)$ : $\pi_{1}(S) \rightarrow \operatorname{PSL}(d ; \mathbb{R})$.

Definition 10. A component of $\operatorname{Hom}\left(\pi_{1}(S), \operatorname{PSL}(d ; \mathbb{R})\right)$ / $\operatorname{PGL}(d ; \mathbb{R})$ is Hitchin provided it contains a representation $\tau_{d}(\rho)$ for $\rho \in \operatorname{Hom}_{d f}\left(\pi_{1}(S)\right.$, PSL $\left.(2 ; \mathbb{R})\right)$. 
Each Hitchin component contains an image of $\mathcal{T}$, called the Fuchsian locus, induced by the mapping $\tau_{d}$.

Labourie developed an in-depth geometric description of Hitchin representations by analyzing the group actions on an appropriate limit set. Sambarino and then more generally Bridgeman-Canary-Labourie-Sambarino defined a topologically transitive Anosov-type flow with closed orbit periods given by logarithms of the maximal eigenvalues of $\operatorname{PSL}(d ; \mathbb{R})$ elements. The authors further established that the mapping to $\mathcal{P}$ is analytic and the essential fact that vanishing of all infinitesimal variations of maximal eigenvalues implies a trivial variation. Their results are combined in the following.

Theorem $11([1,2])$. On a Hitchin component the pressure metric is positive definite and MCG invariant. On a Fuchsian locus the pressure metric restricts to the Weil-Petersson metric.

\section{The Mirzakhani Recursion}

Mirzakhani answered how to integrate over the moduli space [6]. The method begins with a generalization of McShane's length identity. Then start with the identity and replace the sums over topological types with sums over translates of an $M C G$ fundamental domain. The result is a recursion based on summing and integrating over tailoring data for attaching a pair of pants. The resulting integrals are elementary.

We consider surfaces with geodesic boundaries. Introduce the elementary functions

$$
\mathcal{D}(x, y, z)=2 \log \left(\frac{e^{\frac{x}{2}}+e^{\frac{y+z}{2}}}{e^{\frac{-x}{2}}+e^{\frac{y+z}{2}}}\right)
$$

and

$$
\mathcal{R}(x, y, z)=x-\log \left(\frac{\cosh \frac{y}{2}+\cosh \frac{x+z}{2}}{\cosh \frac{y}{2}+\cosh \frac{x-z}{2}}\right) .
$$

For a hyperbolic genus one surface $S$ with geodesic boundary of length $L$, McShane found the identity for geodesic lengths:

$$
L=\sum_{\alpha \text { simple }} \mathcal{D}\left(L, \ell_{\alpha}, \ell_{\alpha}\right)
$$

The boundary length has an exact relation to the lengths of all the simple closed geodesics. The identity comes from an analysis of geodesics perpendicular to the boundary. For the sake of exposition, we overlook the role of the elliptic involution in the following. The set of free homotopy classes of simple closed curves on a torus forms a single $M C G$ orbit. The orbit is enumerated as $\{h(\alpha) \mid h \in$ $M C G / \operatorname{Stab}(\alpha)\}$. The stabilizer $\operatorname{Stab}(\alpha)$ of $\alpha$ is generated by a Dehn twist about $\alpha$. Mapping classes act on geodesic lengths by permuting labels: for $h \in M C G, \ell_{\alpha} \circ h^{-1}=$ $\ell_{h(\alpha)}$. The key observation is that the sum in McShane's identity can be written as a sum over translates of an $M C G$ fundamental domain to obtain the simpler fundamental domain for $\operatorname{Stab}(\alpha)$.

So we write McShane's identity as

$$
L=\sum_{h \in M C G / \operatorname{Stab}(\alpha)} \mathcal{D}\left(L, \ell_{\alpha} \circ h^{-1}, \ell_{\alpha} \circ h^{-1}\right) .
$$

Writing $\mathcal{T}(L)$ for Teichmüller space and $\omega$ for the area element, we integrate over $\mathcal{T}(L) / M C G$ to find

$$
\begin{aligned}
& L V(L) \\
& =\int_{\mathcal{T}(L) / M C G} \sum_{h \in M C G / \operatorname{Stab}(\alpha)} \mathcal{D}\left(L, \ell_{\alpha} \circ h^{-1}, \ell_{\alpha} \circ h^{-1}\right) \omega,
\end{aligned}
$$

writing $V(L)$ for the volume. The change of variables $p=$ $h(q)$ and the formula $\omega=\frac{1}{2} d \ell \wedge d \tau$ gives

$$
\begin{aligned}
=\frac{1}{2} \sum_{h \in M C G / \operatorname{Stab}(\alpha)} & \int_{h(\mathcal{T}(L) / M C G)} \mathcal{D}\left(L, \ell_{\alpha}, \ell_{\alpha}\right) d \tau d \ell \\
& =\frac{1}{2} \int_{\mathcal{T}(L) / \operatorname{Stab}(\alpha)} \mathcal{D}\left(L, \ell_{\alpha}, \ell_{\alpha}\right) d \tau d \ell
\end{aligned}
$$

and use the $\operatorname{Stab}(\alpha)$ fundamental domain to obtain

$$
\frac{1}{2} \int_{0}^{\infty} \int_{0}^{\ell} \mathcal{D}(L, \ell, \ell) d \tau d \ell .
$$

To simplify the integral we form the $L$ partial derivative to find

$$
\frac{\partial}{\partial L} L V(L)=\int_{0}^{\infty} \frac{1}{1+e^{\ell+\frac{L}{2}}}+\frac{1}{1+e^{\ell-\frac{L}{2}}} \ell d \ell=\frac{\pi^{2}}{6}+\frac{L^{2}}{8} .
$$

The volume formula $V(L)=\frac{\pi^{2}}{6}+\frac{L^{2}}{24}$ results. We discuss the general volume formula below. The general recursion is based on a general length identity also proved by analyzing geodesics perpendicular to the boundary.

The Mirzakhani-McShane Identity ([6]). For a hyperbolic surface $S$ with $n$ boundaries $\beta_{j}$ with lengths $L_{j}$,

$$
L_{1}=\sum_{\alpha_{1}, \alpha_{2}} \mathcal{D}\left(L_{1}, \ell_{\alpha_{1}}, \ell_{\alpha_{2}}\right)+\sum_{j=2}^{n} \sum_{\alpha} \mathcal{R}\left(L_{1}, L_{j}, \ell_{\alpha}\right),
$$

where the first sum is over all unordered pairs of simple closed geodesics with $\beta_{1}, \alpha_{1}, \alpha_{2}$ bounding an embedded pair of pants, and the double sum is over simple closed geodesics with $\beta_{1}, \beta_{j}, \alpha$ bounding an embedded pair of pants.

For each sum the topology of the complement of the pair of pants determines the tailoring modulo homeomorphisms. The general recursion comes from summing and integrating over the tailoring data for attaching a pair of pants to surfaces of smaller topological type. 


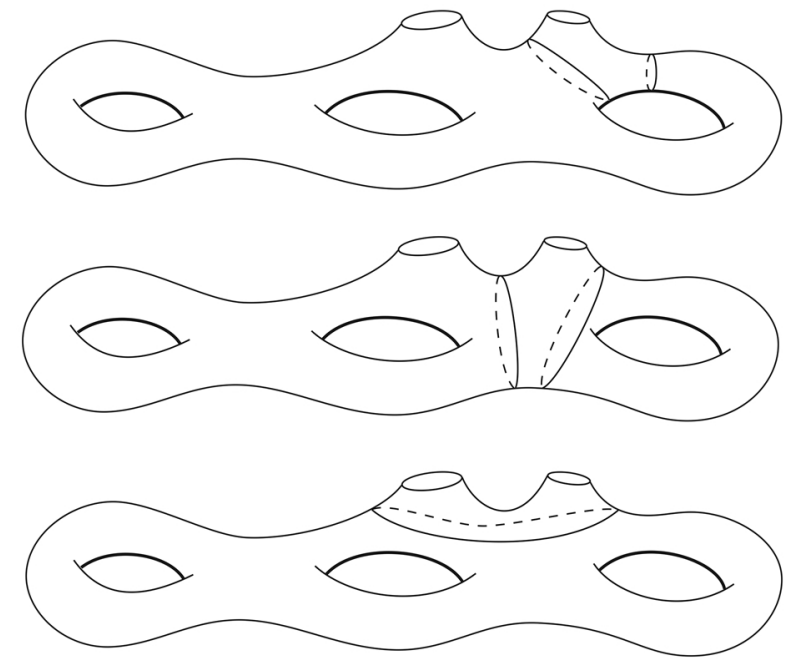

Figure 5. The three basic cases for the Mirzakhani-McShane Identity.

\section{Volumes, Counting Geodesics, and Random Surfaces}

Mirzakhani applied her integration scheme to four applications. In the first, she developed WP volume formulas for the moduli spaces of hyperbolic surfaces with geodesic boundaries of prescribed lengths [6]. In the second, she combined her formulas with symplectic reduction to show that the tautological intersection numbers for the moduli spaces satisfy the KdV recursion hierarchy-a solution of the Witten 2d-gravity conjecture [7]. Her solution came after Kontsevich's and Okounkov-Pandharipande's solutions. In the third, she obtained the asymptotic count by length for simple closed geodesics [8]. In the fourth, joint with Petri, the authors showed that on average the frequencies for collections of lengths of geodesics lying in prescribed disjoint intervals is approximated by independent Poisson random variables [10]. Expositions of the second and third applications are presented in our Park City lectures [12] and CBMS lectures [11].

The Teichmüller and moduli spaces of hyperbolic surfaces with geodesic boundaries are parameterized by Fenchel-Nielsen coordinates with symplectic forms given by a counterpart of Theorem 4 . The moduli space WP volumes are polynomials in boundary lengths.

Theorem 12 (WP volume [6, 7]). The volume polynomials are determined recursively from the volume polynomials of smaller total degree. The volume $V_{g, n}\left(L_{1}, \ldots, L_{n}\right)$ of the moduli space of genus $g, n$ boundaries, hyperbolic surfaces with boundary lengths $L=\left(L_{1}, \ldots, L_{n}\right)$ is a polynomial

$$
V_{g, n}(L)=\sum_{\substack{\alpha \\|\alpha| \leq 3 g-3+n}} C_{\alpha} L^{2 \alpha}
$$

for multi-indices $\alpha=\left(\alpha_{1}, \ldots, \alpha_{n}\right)$ and where $C_{\alpha}>0$ lies in $\pi^{6 g-6+2 n-2|\alpha|} \mathbb{Q}$. The coefficients are the intersection numbers for the Chern classes of the $\kappa_{1}$ and $\psi$ line bundles.

The volume is the $\kappa_{1}$ self-intersection number. The polynomial behavior was already a new result. MirzakhaniZograf further analyzed the recursion to obtain a complete asymptotic expansion for the volume, answering a longstanding question. The volume, now for the moduli space of genus $g$ surfaces with $n$ cusps, is given as

$$
\begin{aligned}
V_{g, n}=C \frac{(2 g-3+n) !\left(4 \pi^{2}\right)^{2 g-3+n}}{\sqrt{g}} & \\
& \left(1+\frac{c_{n}^{(1)}}{g}+\cdots+\frac{c_{n}^{(k)}}{g^{k}}+O\left(\frac{1}{g^{k+1}}\right)\right)
\end{aligned}
$$

for a universal constant $C$ and effectively computable coefficients.

Riven first observed that the count of simple geodesics by length has polynomial magnitude. Mirzakhani applied her recursion to obtain formulas with explicit constants for the asymptotic count. She then extended the results to include nonsimple geodesics. More recently ErlandssonSouto established the general result by synthetic geometrystyle arguments.

A multicurve is a finite formal sum $\gamma=\sum_{i} a_{i} \gamma_{i}$ of pairwise, nonnull homotopic, nonhomotopic, closed curves with positive coefficients. The length $e_{\gamma}(S)$ for the hyperbolic structure $S$ is the weighted sum of geodesic lengths for $S$. A multicurve is simple if all the component geodesics are simple. Two multicurves $\gamma, \gamma^{\prime}$ are of the same type if they belong to the same mapping class group orbit-so $\gamma^{\prime}=h(\gamma)$ for $h \in M C G$. The Mirzakhani and Erlandsson-Souto result is as follows.

Theorem $13([4,8])$. For any multicurve $\gamma$ on $S$,

$$
\lim _{L \rightarrow \infty} \frac{\#\left\{\gamma^{\prime} \text { of type } \gamma \text { and } e_{\gamma^{\prime}}(S) \leq L\right\}}{L^{6 g-6+2 n}}=\frac{c(\gamma) B(S)}{V_{g, n}},
$$

where $c(\gamma)$ is rational depending only on the topology of $\gamma$ and $B(S)$ is the Thurston volume for $S$.

The Erlandsson-Souto analysis includes geodesics with bounded self-intersections lying in narrow channels. They combine with an analysis of the action of $M C G$ on the space of measured geodesic laminations, including $\mathrm{Ma}$ sur's ergodicity theorem. The latter is also an ingredient for Mirzakhani. The polynomial growth for geodesics of fixed type contrasts sharply with the exponential growth $e^{L} / L$ for all geodesics. This mirrors the contrast between the narrow channels for geodesics with bounds on the number of selfintersections and the equidistribution for all geodesics.

What is a random hyperbolic surface? Brooks-Makover used random 3-regular graphs with edges oriented around vertices to describe triangulations on random hyperbolic 
surfaces. Mirzakhani considered random hyperbolic surfaces relative to WP volume. Invariants such as the systole (length of shortest closed geodesic), radius of the largest embedded ball, diameter of the surface, number of eigenvalues of the Laplace-Beltrami operator in a given finite interval, or the number of primitive geodesic lengths in a given finite interval are all examples of random variables on the moduli space $\mathcal{M}_{g}$. What are the expected values and distributions of these random variables?

Mirzakhani's most specific result, joint with Petri, gives the distribution of geodesic lengths in intervals. For an interval $[a, b] \subset \mathbb{R}_{+}$and $S \in \mathcal{M}_{g}$, let $N_{g,[a, b]}(S)$ be the number of closed geodesic lengths of $S$ in the interval $[a, b]$. $N_{g,[a, b]}$ is a random variable with values in the natural numbers $\mathbb{N}$. A random variable $Z$ on a probability space is Poisson distributed with positive mean $\lambda$, provided its probabilities are given as $\mathbb{P}[Z=k]=\lambda^{k} e^{-\lambda} / k$ ! for all $k \in \mathbb{N}$. MirzakhaniPetri showed for large genera the length-counting random variables $N_{g,[a, b]}$ limit to independent Poisson distributed random variables.

Theorem 14 ([10]). Let $\left[a_{1}, b_{1}\right],\left[a_{2}, b_{2}\right], \ldots,\left[a_{k}, b_{k}\right] \subset \mathbb{R}_{+}$ be disjoint intervals. Then as $g$ tends to infinity, the vector of random variables

$$
\left(N_{g,\left[a_{1}, b_{1}\right]}, \ldots, N_{g,\left[a_{k}, b_{k}\right]}\right)
$$

converges jointly in distribution to a vector of independent Poisson distributed variables with means

$$
\lambda_{\left[a_{i}, b_{i}\right]}=\int_{a_{i}}^{b_{i}} \frac{\cosh t-1}{t} d t
$$

for $i=1, \ldots, k$.

The quantity $e^{-\lambda_{[0, R]}}$ is the probability that all lengths lie in the interval $[R, \infty)$. A first application is that the probablity of the systole being at most $\epsilon$ is computed from the complement

$$
\lim _{g \rightarrow \infty} \mathbb{P}_{g}[\operatorname{sys}(S) \leq \epsilon]=1-e^{-\lambda_{[0, \epsilon]}} \sim 1-e^{-\epsilon^{2} / 4} \sim \frac{\epsilon^{2}}{4} .
$$

Similarly $\epsilon^{4} / 16$ is the probability of at least two geodesic lengths less than $\epsilon$. So in large genus there is a positive probability of a hyperbolic surface having small lengths. The authors also apply their result to evaluate the limiting expected value (the average) of the systole

$$
\lim _{g \rightarrow \infty} \mathbb{E}_{g}[s y s]=\int_{0}^{R} e^{-\lambda_{[0, R]}} d R=1.61498 \ldots .
$$

Mirzakhani studies expected values of various random variables, showing that the separating systole (length of the shortest separating geodesic) satisfies

$$
\mathbb{E}_{g}[\text { sepsys }] \asymp \log g,
$$

the surface diameter satisfies

$$
\mathbb{E}_{g}[\sqrt{\text { diameter }}] \asymp \sqrt{\log g},
$$

and the injectivity radius (radius of the largest embedded ball) satisfies

$$
\mathbb{E}_{g}[i n j] \asymp \log g,
$$

where $\asymp$ connotes bounded above and below with constants independent of genus.

Most recently teams of authors are revisiting bounds for the first eigenvalue $\lambda_{1}$ of the Laplace-Beltrami operator for large genus hyperbolic surfaces. Magee-Naud-Puder show that asymptotically almost surely in the tower of coverings of a given compact surface, $\lambda_{1}$ is at least 3/16. LipnowskiWright and Wu-Xue separately show that

$$
\lim _{g \rightarrow \infty} \mathbb{P}_{g}\left[\lambda_{1}(S) \geq \frac{3}{16}-\epsilon\right]=1 \text {. }
$$

ACKNOWLEDGMENTS. It is my pleasure to thank Martin Bridgeman, Richard Canary, Viveka Erlandsson, and Alexander Wright for their suggestions.

\section{References}

[1] Martin Bridgeman, Richard Canary, François Labourie, and Andres Sambarino, The pressure metric for Anosov representations, Geom. Funct. Anal. 25 (2015), no. 4, 1089-1179, DOI 10.1007/s00039-015-0333-8, MR3385630

[2] Martin Bridgeman, Richard Canary, and Andrés Sambarino, An introduction to pressure metrics for higher Teichmüller spaces, Ergodic Theory Dynam. Systems 38 (2018), no. 6, 2001-2035, DOI 10.1017/etds.2016.111. MR3833339

[3] Peter Buser, Geometry and spectra of compact Riemann surfaces, Progress in Mathematics, vol. 106, Birkhäuser Boston, Inc., Boston, MA, 1992. MR1183224

[4] Viveka Erlandsson and Juan Souto, Counting curves in hyperbolic surfaces, Geom. Funct. Anal. 26 (2016), no. 3, 729777, DOI 10.1007/s00039-016-0374-7. MR3540452

[5] John Hamal Hubbard, Teichmüller theory and applications to geometry, topology, and dynamics. Vol. 1: Teichmüller theory, Matrix Editions, Ithaca, NY, 2006. With contributions by Adrien Douady, William Dunbar, Roland Roeder, Sylvain Bonnot, David Brown, Allen Hatcher, Chris Hruska and Sudeb Mitra; With forewords by William Thurston and Clifford Earle. MR2245223

[6] Maryam Mirzakhani, Simple geodesics and Weil-Petersson volumes of moduli spaces of bordered Riemann surfaces, Invent. Math. 167 (2007), no. 1, 179-222, DOI 10.1007/s00222006-0013-2. MR2264808

[7] Maryam Mirzakhani, Weil-Petersson volumes and intersection theory on the moduli space of curves, J. Amer. Math. Soc. 20 (2007), no. 1, 1-23, DOI 10.1090/S0894-0347-0600526-1. MR2257394.

[8 Maryam Mirzakhani, Growth of the number of simple closed geodesics on hyperbolic surfaces, Ann. of Math. (2) 168 (2008), no. 1, 97-125, DOI $10.4007 /$ annals.2008.168.97 MR2415399

[9] Maryam Mirzakhani, Growth of Weil-Petersson volumes and random hyperbolic surfaces of large genus, J. Differential Geom. 94 (2013), no. 2, 267-300. MR3080483 
[10] Maryam Mirzakhani and Bram Petri, Lengths of closed geodesics on random surfaces of large genus, Comment. Math. Helv. 94 (2019), no. 4, 869-889, DOI 10.4171/cmh/477. MR4046008

[11] Scott A. Wolpert, Families of Riemann surfaces and WeilPetersson geometry, CBMS Regional Conference Series in Mathematics, vol. 113, Published for the Conference Board of the Mathematical Sciences, Washington, DC; by the American Mathematical Society, Providence, RI, 2010, DOI 10.1090/cbms/113. MR2641916

[12] Scott A. Wolpert, Mirzakhani's volume recursion and approach for the Witten-Kontsevich theorem on moduli tautological intersection numbers, Moduli spaces of Riemann surfaces, IAS/Park City Math. Ser., vol. 20, Amer. Math. Soc., Providence, RI, 2013, pp. 221-266, DOI 10.1090/pcms/020/08. MR3114687

[13] Scott Wolpert, On the Weil-Petersson geometry of the moduli space of curves, Amer. J. Math. 107 (1985), no. 4, 969997, DOI 10.2307/2374363. MR796909

[14] Scott A. Wolpert, Thurston's Riemannian metric for Teichmüller space, J. Differential Geom. 23 (1986), no. 2, 143174. MR845703

[15] Alex Wright, A tour through Mirzakhani's work on moduli spaces of Riemann surfaces, Bull. Amer. Math. Soc. (N.S.) 57 (2020), no. 3, 359-408, DOI 10.1090/bull/1687. MR4108090

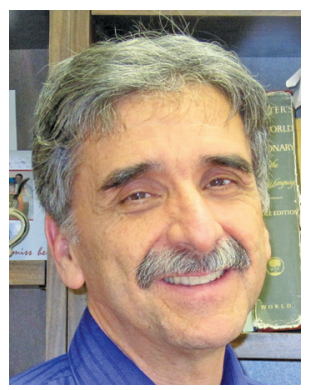

Scott A. Wolpert

\section{Credits}

Opening image is courtesy of Kilom691 and Alexander Hulpke via Wikimedia Commons. Licensed under the Creative Commons Attribution-Share Alike 4.0 International license.

Figures 1, 2, 4, and 5 and photo of Scott A. Wolpert are courtesy of Scott A. Wolpert.

Figure 3 is courtesy of donald_gruener via Getty.

\section{Give the Gift of AMS}

\section{MEMBERSHIP}

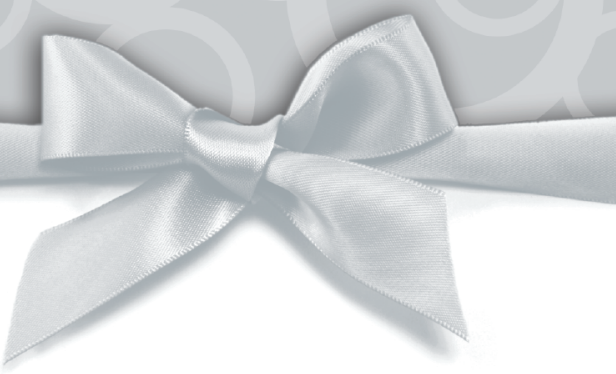

Surprise someone with the gift of connection to the mathematical community. Your purchase of an AMS Membership supports the AMS in its mission to further mathematical research, scholarship, professionalism, education, and awareness. It is a gift that keeps on giving.

There are three easy steps to make your gift membership purchase:

Visit www.ams.org/membership/ member-application-18.pdf to download the AMS Membership application.

Complete the application for the gift recipient.

Mail the completed application with payment. Include a simple letter that indicates the membership is a gift.

Mail to:

American Mathematical Society Sales and Member Services Department 201 Charles Street

Providence, RI 02904-2213 USA

If you have questions, please contact the Sales and Member Services Department at: cust-serv@ams.org or 800.321.4267.

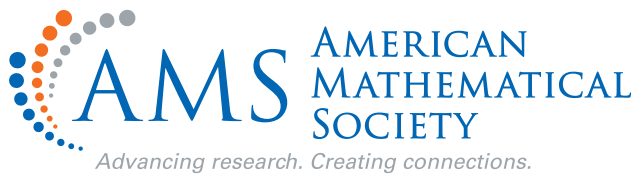

